\title{
A joint approach to treating dental phobia: a re-evaluation of a collaboration between community dental services and specialist psychotherapy services ten years on
}

\author{
J. G. Davies, ${ }^{1}$ K. I. Wilson ${ }^{2}$ and A. L. Clements $^{3}$ \\ VERIFIABLE CPD PAPER
}

Objective To audit the records of a group of patients who had previously benefited from cognitive behavioural therapy (CBT) for dental phobia. Aim To ascertain if they had returned to the use of intravenous (IV) sedation to facilitate dental treatment. Ten years ago these patients were routinely requiring IV sedation to facilitate dental treatment due to severe dental phobia. Method Sixty patients entered the original pilot project. Of those, 30 were offered CBT and 21 attended. Twenty of those patients (95.2\%) were subsequently able to have dental treatment without IV sedation. In this follow-up study the electronic records of 19 of the 20 patients who had originally been successful with CBT were re-audited. Our purpose was to see if there was any record of subsequent IV sedation administration in the intervening ten years. Results Of the 19 successful CBT patients available to follow-up, 100\% had not received IV sedation since the study ten years ago. This may suggest the initial benefit of CBT has endured over the ten-year period. Conclusion This study indicates that the use of CBT for patients with dental phobia proves beneficial not only in the initial treatment but that the benefits may endure over time. This results in a significant reduction in health risks to the patient from repeated IV sedation. It may also translate into significant financial savings for dental care providers. Our evidence for CBT as treatment for dental phobia suggests dental services should be implementing this approach now rather than pursuing further research.

\section{INTRODUCTION}

Dental anxiety and related problems affect a significant proportion of the population $^{1}$ and patients with dental phobia prove to be extremely consuming of time and resources.

The traditional approach to this group has been to use IV sedation to facilitate treatment. Recently questions have been raised about this strategy. The economic and human cost of anaesthetic related death and morbidity have resulted in the directive that from 31 December 2001 general anaesthesia for dental treatment

"Consultant Adult Psychotherapist/Clinical Lead for Cognitive Behavioural Psychotherapy, Sheffield Health and Social Care Foundation Trust; ${ }^{2}$ Consultant in Special Care Dentistry, Heart of Birmingham Teaching PCT, Aston Health Centre; ${ }^{3}$ Higher Specialty Trainee in Adult Psychiatry, Sheffield Health and Social Care Foundation Trust

${ }^{*}$ Correspondence to: Mr John Davies

Email: john.davies@shsc.nhs.uk

\section{Refereed Paper}

Accepted 10 June 2011

DOI:10.1038/sj.bdj.2011.674

${ }^{\text {QB }}$ ritish Dental Journal 2011; 211: 159-162 should only be administered in a hospital setting with critical care facilities. ${ }^{2}$

There is significant evidence that CBT interventions are effective in the treatment of all types of phobias and anxieties including dental. ${ }^{1,3,4}$ Research has also shown that even brief CBT interventions can have significant positive effect, ${ }^{5}$ in particular that such intervention will produce a significant and sustained reduction in negative cognitions towards dental care and its related anxiety. ${ }^{6,7}$

In practice a reduction in fear and anxiety leads to a reduction in the need for IV sedation in phobic patients. It has been suggested that a large proportion of patients presenting with dental anxieties can be treated solely with psychological treatments such as behavioural management techniques. ${ }^{8}$

In a recent study by Wooley ${ }^{9}$ they found that in a 12 month period none of the referrers requested behavioural management techniques as a method of anxiety management. This was echoed by McGoldrick et al.: '...in spite of the efficacy of psychological treatments for dental anxiety, primary and secondary care dentists appear not to be suggesting or promoting their use for patients with dental anxiety...'

Sheffield's Salaried Primary Dental Care Service (formerly Community Dental Service) has operated a special service for patients with dental phobia since 1992. Provided by a group of experienced dentists the service promotes basic anxiety management techniques and gives advice regarding the risks of IV sedation. While the service has been generally successful, a number of severely phobic patients have been unable to complete treatment without recourse to the use of IV sedation, and dependence on specialised dental services for future treatment.

Given the evidence for the efficacy of a CBT approach in the treatment of anxiety and phobias a small pilot project comparing community dentistry in combination with brief CBT versus treatment as usual 


\begin{tabular}{|c|c|c|c|}
\hline Attendance & Dentist & Psychotherapist & Chi Squared Test \\
\hline Attended & 17 & 21 & \multirow{3}{*}{$\begin{array}{l}p<0.4 \\
\text { Not significant }\end{array}$} \\
\hline Did not attend & 13 & 9 & \\
\hline Total & 30 & 30 & \\
\hline
\end{tabular}

Table 2 Initial rates of IV sedation

\begin{tabular}{|l|l|l|l} 
Sedation & Dentist & Psychotherapist & Chi Squared Test \\
\hline No sedation & 5 & 20 & \\
\cline { 1 - 2 } IV sedation & 12 & 1 & $\begin{array}{l}\text { S }<0.001 \\
\text { Significant }\end{array}$ \\
\cline { 1 - 2 } Total & 17 & 21 & \\
\hline
\end{tabular}

was conducted. It was predicted that CBT for dental phobia would reduce the use of IV sedation during dental treatment for those patients who relied on IV sedation to manage their dental fears. The initial pilot results were positive and the method and results are outlined later.

Although there is already a body of published evidence that CBT intervention will be successful in the initial management of dental anxieties there is little long term data. This follow up evaluation specifically addresses the question: does the beneficial effect of CBT endure over time?

\section{MATERIALS AND METHODS}

The initial pilot utilised two clinicians: an experienced Cognitive Behavioural Psychotherapist and a Senior Community Dentist. A random sample of 60 patients referred to the existing specialist dental phobia service was allocated on an alternate basis to either standard treatment or standard treatment with additional CBT. An example of the CBT intervention is described by Wilson and Davies in a previously published single case study and is quoted below:

'The approach focused on a cognitive behavioural approach adapted from de Jongh et al., "One session treatment for dental phobics" and 0st, "Treatment for specific phobia". This approach aims to help the patient understand how their fears have been maintained and gives pragmatic solutions to reverse their patterns of behaviour. The initial session focused on a clear formulation as to how the problems had evolved and subsequently process the patient was given information which explained how, in general, anxiety operated and affected thought, behaviour and physical reactions. This understanding formed the basis of their psychological education and allowed the techniques to undermine their fear. Cognitive or thought-based interventions are based on the patient using this acquired information about the nature of fear to test and challenge their interpretations of fear events (an evidential approach). The patient was then introduced to the principles of exposure and how exposure-based therapies aim to reduce fears by a reduction in avoidance behaviour, and their re-evaluation of thinking associated with fear. ${ }^{10}$

The patients were all secondary referrals from other healthcare professionals. The commonest source of referral was the Charles Clifford Dental Hospital Casualty Department, followed by general dental practitioners and then general medical practitioners. No formal psychiatric assessment or diagnosis was made at the time of initial referral.

Patients allocated to the dentist were seen using the existing standard protocol. This consisted of advice regarding the risks of IV sedation, anxiety management leaflets, and the enhanced consultation skills of an experienced dentist.

Patients allocated to the psychotherapist were sent a letter explaining that they would be seen initially in the Department of Psychotherapy, a non-dentistry environment and would then subsequently receive their dental treatment as usual. been maintained. To further reinforce this
Table 3 Ten year follow up IV sedation rates

\begin{tabular}{l|l} 
Sedation & Psychotherapist \\
\hline No sedation & 19 \\
\hline IV sedation & 0 \\
\hline Total & 19 \\
\hline
\end{tabular}

In both cases patients were given one initial appointment. Should they fail to attend they were sent a standard letter asking them to contact the appropriate clinic should they wish for a further appointment.

The current audit was of the 20 patients who received the CBT intervention ten years ago and were able to have their subsequent dental treatment without IV sedation. The administration of IV sedation in Sheffield is primarily via the community dental services and dental hospital therefore enabling the patient's use of IV sedation to be assessed via an audit of the electronic records.

The ten year follow up data for the patient cohort which was allocated to the treatment as usual group, and consequently did not receive CBT, is not available for comparison.

\section{RESULTS}

Of the 60 patients referred, 38 patients attended for treatment. Of the patients who attended there was no statistical difference between those attending the dentist versus the psychotherapist (Table 1). However, there was a significant difference in the numbers of patients requiring IV sedation to complete their dental treatment. $70.6 \%$ of patients allocated to treatment as usual required IV sedation whereas $4.8 \%$ of the patients allocated to the psychotherapist required IV sedation (Table 2 ).

We were able to follow up 19 of the original patients allocated to CBT. One patient had moved out of city and we were unable to follow up their records. None of the other 19 CBT patients, who were successful in having dental treatment without IV sedation ten years ago, had required subsequent IV sedation (Table 3). Three of the patients had required the use of inhalational sedation (nitrous oxide gas) to manage a dental procedure and one used hypnosis. Six of the 19 patients had had no contact with dental services at all over the past ten years. However, 
13 had continued to receive dental treatments including scale and polish, fillings, extractions, impressions, and temporary and permanent crowns. Two of the original psychotherapy patients had been discharged as they felt able to return to the primary general dental service for their ongoing management (Table 4).

We can be reasonably confident that $68.4 \%$ of the original psychotherapy patients have experienced a sustained improvement in their phobia and have continued to receive a variety of dental interventions without recourse to IV sedation. We cannot be certain as to the progress of the six patients who have had no contact. They too may have a sustained benefit but have not required any dental treatment. We must be open to the possibility that their dental phobia reasserted itself and they have been avoiding contact with dental services.

Ten percent (two) of the original cohort of 20 who successfully completed the CBT arm required no further input from the community dental services following the initial intervention, and have not had further recourse for IV sedation from secondary dental services in Sheffield.

\section{DISCUSSION}

Our follow up evaluation appears to indicate that the initial recovery the dental phobia patients allocated to CBT made has endured over a ten-year period, with none returning to the use of IV sedation.

A previous study by Kvale et al. ${ }^{11}$ concluded that patients who had received a behavioural intervention for dental phobia showed a $46.5 \%$ continued improvement up to four years after treatment. Our reevaluation appears to indicate a greater degree of improvement (95.2\%) with the initial psychological intervention and at long term follow up (68.4\%). Our result may be distorted due to the small numbers in our study and other non-specific factors such as individual patient's motivation to attend and change. In our initial pilot patients who had been offered a psychological intervention could choose to have treatment as usual. This may account for the higher than predicted response to treatment as those attending for therapy had already demonstrated motivation to engage in psychological treatment by choosing to attend.

\section{Table 4 Detailed ten year follow up data}

\begin{tabular}{l|l}
\hline $\begin{array}{l}\text { Patients successful with } \\
\text { CBT during initial study }\end{array}$ & Dental treatment received in intervening ten years \\
\hline 1 & Fillings and referred back to General Dental Service \\
\hline 2 & Fillings, scale and polish and referred back to General Dental Service \\
\hline 3 & Fillings, extractions, scale and polish \\
\hline 4 & Crown, scale and polish, fillings \\
\hline 5 & Fillings, extractions \\
\hline 6 & Denture, extractions, fillings \\
\hline 7 & Temporary and permanent crowns \\
\hline 8 & Extractions \\
\hline 9 & Dentures, extractions, scale and polish, impressions \\
\hline 10 & Fillings under hypnosis \\
\hline 11 & Fillings, extractions with gas \\
\hline 12 & Fillings, crowns with gas \\
\hline 13 & Filling, extraction and dentures with gas \\
\hline 14 & No contact \\
\hline 15 & No contact \\
\hline 16 & No contact \\
\hline 17 & No contact \\
\hline 18 & No contact \\
\hline 19 & No contact \\
\hline 10 & \\
\hline 10 &
\end{tabular}

Although caution needs to be taken when interpreting our data, comparative data from other studies based on behavioural management techniques ${ }^{8}$ demonstrate that psychological techniques are at least as effective or show little difference when compared with traditional use of IV sedation.

Previous audits of patients referred to secondary care clinics ${ }^{1}$ have found that although patients are routinely identified as having dental related anxieties or fears, they are rarely offered an alternative to IV sedation. A recent study by Boyle et al. ${ }^{12}$ again reported that although patients had been identified as being dentally anxious the main intervention offered had been IV sedation as means to manage their dental treatment.

While there is specific evidence for CBT as psychological treatment for dental phobia, the evidence in the psychological literature that CBT interventions are successful in the management of other anxiety and phobic related problems is compelling. ${ }^{4}$ There is no theoretical reason why this evidence cannot bolster the case for psychological treatment of dental phobia as the aetiology is broadly similar.

However, access to psychological treatments within dental services remains limited even though it has been identified that "psychological interventions are a feasible option and some patients prefer it when given a choice.' ${ }^{13}$

\section{CONCLUSIONS}

This evaluation, although having flaws as outlined in the discussion, is still consistent with the past literature regarding the feasibility and success of psychological interventions for dental phobia. It adds and enhances this body of literature by indicating the possibility of a longer lasting effect from a psychological intervention than previously recognised.

The initial comparison also indicates that $10 \%$ of patients who have previously been dependent on Community Dental Services move back to general dental practitioners (GDPs) if offered an appropriate psychological intervention. This would be an area 
for further exploration given the difficulties in moving this dependent group of patients from Community Dental Services to GDPs. It could be speculated that if such a service were routinely offered that this problem may diminish, but this has not been the main focus of this re-evaluation.

Our study suggests that failing to develop services which include CBT as an intervention choice for patients may be denying individuals a therapy that not only manages their difficulties within the immediate time, but may have long lasting health benefits. We have not examined the economic benefit of providing a psychotherapy service; but our findings are likely to be of interest to health care providers. The potential costs to health services of individuals who become dependent on IV sedation to manage their fears and the potential morbidity and mortality ${ }^{14}$ need to be considered.

The service in Sheffield has primarily focused on referrals of the most severely phobic patients to psychological services. However, in line with other NICE guidance for anxiety we suggest a stepped model could be developed and implemented. For example the appropriate use of computerised CBT as an initial intervention and the use of other allied dental professionals, such as hygienist or nurses, to deliver CBT based interventions. This could significantly reduce the burden of dental phobia now and in the future, the rationale being that anxiety problems identified and treated early will be less likely to develop into severe phobias in the future. A stepped care approach would also reflect the current pragmatic reality in the world of mental health where Improving Access to Psychological Therapies (IAPT) has placed an increasing focus on widening the access to evidence-based psychological interventions

Given the evidence within the wider field of mental health, there is a compelling argument that the focus for dental services should be on the development of models of delivery, care pathways and education of dental health professionals in CBT approaches. In their report prepared for the Centre for Economic Performance, related to the cost benefit analysis of providing psychological therapy Clark, Knapp, Layard and Mayraz reported that their findings 'strongly reinforce the humanitarian case for implementing the NICE Guidelines ${ }^{15}$ which clearly advocate a psychological approach to anxiety and related problems. Therefore why should this not be the case for the dental patients who are currently routinely denied an evidencebased intervention for their difficulties?

1. McGoldrick P, Levitt J, deJong A, Mason A, Evans D. Referrals to a secondary care dental clinic for anxious adult patients: implications for treatment. Br Dent J 2001; 191: 686-688.

2. Layard R, Clark D, Knapp M, Mayraz G. Cost-benefit analysis of psychological therapy, 2007. Centre for Economic Performance, London School of Economics and Political Science Publication.

3. National Institute for Health and Clinical Excellence. Generalised anxiety disorder and panic disorder (with or without agoraphobia) in adults. Management in primary, secondary and community care. Clinical Guideline 113. London: National Institute for Health and Clinical Excellence, 2011.

5. Roth A, Fonagy P, Parry G. What works for whom? A critical review of psychotherapy research. p119. New York: Guildford Press, 1996.

6. Ost L-G. One session treatment for specific phobias. Behav Res Ther 1989; 27: 1-7.

7. De Jongh A, Muris P, Ter Horst G, Van Zuuren F Schoenmakers N, Makkes P. One session cognitive treatment of dental phobia: preparing dental phobics for treatment by restructuring negative cognitions. Behav Res Ther 1995; 33: 947-954.

8. Hakeberg M, Berggren U, Carlsson S G. Long-term effects on dental care behaviour and dental health after treatments for dental fear. Anesth Prog 1993; 40: 72-77.

9. Aartman H A, de Jongh A, Makkes P C, Hoogstraten J. Behavioral management: treatment modalities in a dental fear clinic and the relation with general psychopathology and oral health variables. Br Dent J 1999 : 186: 467-471.

10. Woolley S M. An audit of referrals to a secondary care sedation unit. Br Dent J 2009; 206: E10.

12. Wilson K I, Davies J G. A joint approach to treating dental phobics between community dental services and specialist psychotherapy services - a single case report. Br Dent J 2001; 190: 431-432.

13. Kvale G, Berggensen U, Milgrom P. Dental fear in adults: a meta-analysis of behavioral interventions. Community Dent Oral Epidemiol 2004; 32: $250-264$.

16. Boyle C A, Newton T, Milgrom P. Who is referred for sedation and why? Br Dent J 2009; 206: E12.

18. Humphris G. Dentally-anxious patients - a study of secondary care referrals in Scotland. Br Dent J 2001; 191: 676 .

19. Department of Health. A conscious decision. A review of the use of general anaesthesia and conscious sedation in primary dental care. London: Department of Health, 2000.

20. Clark D, Knapp M, Layard R, Mayraz G. Cost benefit analysis of psychological therapy. London: Centre for Economic Performance, 2007. Paper no. CEPDP0829. 